\title{
Families of knots for which Morton's inequality is strict
}

\author{
Mark Brittenham and JaCQueline Jensen
}

We describe a procedure for creating infinite families of knots, each having the maximum degree of their HOMFLYPT polynomial strictly less than twice their canonical genus.

\section{Introduction}

Every knot $K$ in the 3 -sphere $S^{3}$ is the boundary of a compact orientable surface $\Sigma \subseteq S^{3}$, known as a Seifert surface for the knot $K$. In fact, in the 1930s Seifert [20] gave an algorithm which, given a diagram of the knot $K$, produces such a surface. The algorithm consists of orienting the knot diagram, breaking each crossing and reconnecting the four ends according to the orientation, without re-introducing a crossing, producing disjoint "Seifert" circles in the projection plane, bounding (after offsetting nested circles) disjoint "Seifert" disks, and then re-introducing the crossings by stitching the disks together with half-twisted bands.

Every knot therefore has infinitely many such "canonical" surfaces (of arbitrarily high genus). The minimum of the genera of the surfaces built by Seifert's algorithm, over all diagrams of the knot $K$, is known as the canonical genus or diagrammatic genus of $K$, denoted $g_{c}(K)$. The minimum genus over all Seifert surfaces, whether built by Seifert's algorithm or not, is known as the genus of $K$, and denoted $g(K)$.

In 1985, shortly after the discovery of the HOMFLYPT polynomial $[5,18]$, Morton [16] showed that the highest degree of the $z$-variable (in his formulation) of the HOMFLYPT polynomial gave a lower bound on $2 g_{c}(K)$; details are outlined below. This was, perhaps, the first piece of information encoded in the HOMFLYPT polynomial to be related to topological information about the knot $K$. Morton's inequality, $M(K)=\operatorname{maxdeg}_{z} P_{K}(v, z) \leq$ $2 g_{c}(K)$, has since been shown to be an equality for many classes of knots. These include all of the knots having 12 or fewer crossings [22], all alternating knots [4,14], and, more generally, all homogeneous knots [3], and the Whitehead doubles of 2-bridge knots [15,24] and alternating arborescent 
knots $[1,10]$. It was not until 1998 and later that knots were found for which Morton's inequality was strict. The first such were found by Stoimenow $[22,23]$ while analyzing the survey of knots through 16 crossings.

In this paper, we show how to use Stoimenow's second collection of examples, or any other example that might be built along the same lines, to build infinite families of knots having $M(K)=\operatorname{maxdeg}_{z} P_{K}(v, z)<2 g_{c}(K)$. In particular, we have the following

Theorem 1.1. Suppose $K$ is a knot with $g(K)=g_{c}(K)$ and $M(K)<2 g_{c}(K)$. Let $D$ be a $g_{c}$-minimizing diagram of $K$, and suppose there is a crossing $c$ in $D$ which bounds a half-twisted band connecting a pair of Seifert disks, and the knot $K^{\prime}$ obtained by changing the crossing $c$ has $g_{c}\left(K^{\prime}\right)<g_{c}(K)$. Then the knots $K_{n}$, bounding the canonical Seifert surfaces $\Sigma_{n}$ obtained by replacing the half-twisted band at the crossing $c$ with $2 n+1$ half-twisted bands in parallel, all joining the same pair of Seifert disks, all satisfy $M\left(K_{n}\right)<$ $2 g_{c}\left(K_{n}\right)=2\left(g_{c}(K)+n\right)$.

The figures in Section 2 should make the construction clear, if the description above did not. We note that the condition $g_{c}\left(K^{\prime}\right)<g_{c}(K)$ simply states that the diagram $D^{\prime}$ obtained from changing the crossing $c$ is not a $g_{c}$-minimizing diagram for $K^{\prime}$; this can often in practice be verified fairly quickly, as our examples below show.

In particular, since, as we shall see, some of Stoimenow's examples satisfy the hypotheses of the theorem, and we only need one to get the process started, we have

Corollary 1.2. There exist knots $K$ with arbitrarily large canonical genus for which $M(K)<2 g_{c}(K)$; in particular, there are infinitely many of them.

\section{Notations and preliminaries}

$K$ will always denote a knot in the 3-sphere $S^{3}, N(K)$ a tubular neighborhood of $K, E(K)=S^{3} \backslash \operatorname{int} N(K)$ the exterior of $K, \Sigma$ a Seifert surface for $K$ (which we treat as embedded in $S^{3}$, or properly embedded in $E(K)$, as needed), and $E(\Sigma)=E(K) \mid \Sigma=E(K) \backslash$ int $N(\Sigma)$ the exterior of $\Sigma$. $E(\Sigma)$ can be thought of as a sutured manifold [6], that is, a compact manifold $M$ with $\partial M=R_{+} \cup R_{-}$, where $R_{+} \cap R_{-}=\gamma$ is a collection of simple closed curves, the sutures. Formally, torus boundary components with no sutures may also be treated as a suture. In our case $\gamma=$ the core of the annulus $\partial E(K) \backslash \partial \Sigma$, cutting $\partial E(\Sigma)$ into two copies of $\Sigma$, pushed off of $\Sigma$ to the +- and --sides. The pair $(E(K), \partial E(K))$ is also an example. 

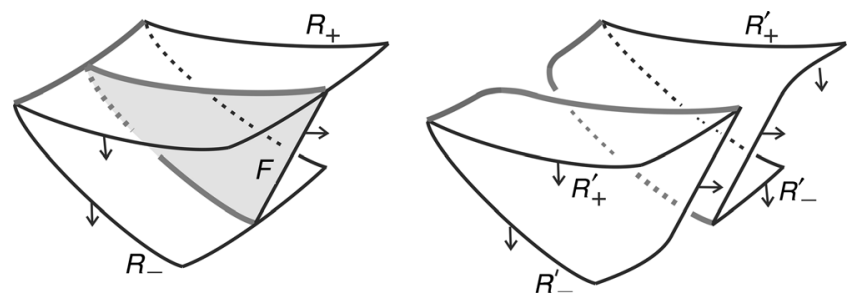

Figure 1: Sutured manifold decomposition.

The theory of sutured manifolds will play a central role to our proofs below; we summarize the main points here. $R_{+}$and $R_{-}$are thought of as having normal orientations, pointing into $M$ along $R_{+}$and out of $M$ along $R_{-}$. Given a properly embedded surface $F \subseteq M$ with a normal orientation and $\partial F$ transverse to $\gamma$, we can decompose $(M, \gamma)$ along $F$ to obtain a new sutured manifold $\left(M \mid F, \gamma^{\prime}\right)$, where the new sutures are constructed by an oriented sum of $\gamma$ and $\partial F$, as in figure 1. A degenerate (but important) example is the decomposition $(E(K), \partial E(K)) \Rightarrow(E(\Sigma), \gamma)$ along $\Sigma$. A sequence of decompositions is a sutured manifold decomposition. A sutured manifold is taut if it admits a sutured manifold decomposition ending with a disjoint union of sutured manifolds of the form $\left(B^{3}, e\right)$, where $e$ is the equatorial circle in the boundary of the 3 -ball $B^{3}$. The sequence of decomposing surfaces used in the decomposition is then called a taut sutured manifold hierarchy. A fundamental theorem of Gabai [6] states that $(E(\Sigma), \gamma)$ admits a taut sutured manifold hierarchy if and only if $\Sigma$ has minimal genus among all Seifert surfaces for $K$. This gives, in principle, an effective way to compute the genus of a knot or link [8]. We will use this technology in Section 2 to compute the genera of our family of knots $K_{n}$.

The HOMFLYPT polynomial $[5,18]$ is a 2 -variable Laurent polynomial defined for any oriented link, and may be thought of as the unique polynomial $P_{K}(v, z)$, defined on oriented link diagrams and invariant under the Reidemeister moves, satisfying $P_{\text {unknot }}(v, z)=1$, and $v^{-1} P_{D_{+}}-v P_{D_{-}}=$ $z P_{D_{0}}$, where $D_{+}, D_{-}, D_{0}$ are diagrams for oriented links which all agree except at one crossing, where they are given as in figure 2. Here we are following the convention in the naming of our variables found in Morton's paper [16].

This skein relation gives an inductive method for computing the polynomial, since one of $D_{+}, D_{-}$will be "closer" than the other to the unlink, in terms of unknotting number, while $D_{0}$ has fewer crossings. It also allows any one of the polynomials to be computed from the other two. We will use 


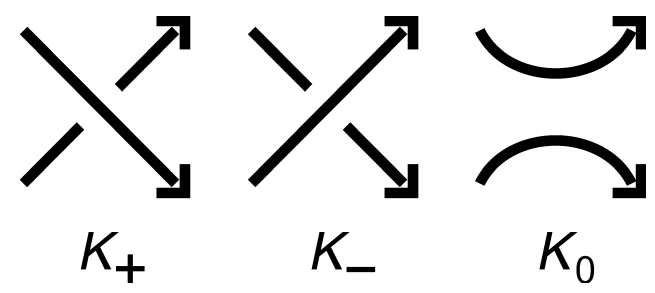

Figure 2: HOMFLYPT polynomial.

this relation in Section 3 to give upper bounds on the $z$-degree $M\left(K_{n}\right)$ of our family of knots $K_{n}$.

\section{The canonical genus via sutured handlebodies}

The proof of our main theorem will be carried out in two steps. In this section, we will show that the knots $K_{n}$ described in the theorem have the canonical genus claimed: $g_{c}\left(K_{n}\right)=g_{c}(K)+n$. In the next section, we will show that the HOMFLYPT polynomials of these knots have $z$-degree $M\left(K_{n}\right)$ strictly lower than twice this.

The basic idea behind Stoimenow's examples, and our argument, is that if $g(K)$ is achieved by a canonical Seifert surface, then this can be detected using sutured manifold theory. In particular, a canonical Seifert surface $\Sigma$ has exterior $E(\Sigma)=E(K) \mid \Sigma$ a handlebody, which we endow with a sutured manifold structure as above. If $(E(\Sigma), \gamma)$ admits a taut sutured manifold hierarchy, then $\Sigma$ is a genus-minimizing, and hence $g_{c}$-minimizing, Seifert surface for $K$. For a sutured handlebody, the simplest hierarchy we can hope for is a disk decomposition, that is, the intermediate decomposing surfaces are (compressing) disks for $E(\Sigma)$.

It is known that not all taut sutured handlebodies are disk decomposable [9], and this phenomenon can even occur for Seifert surface exteriors [2]. But in the case of Stoimenow's examples, a set of decomposing disks is readily available. We show such a set of disks for the first example in figure 3 . The left-hand side of the figure describes a template for constructing the sutured handlebody $(E(\Sigma), \gamma)$ as a standard handlebody, at the right; the dotted line represents the intersection of a vertical plane of the paper running down the middle of the handlebody at the right. "B" marks the back of the figure at right, " $\mathrm{F}$ " the front. Looking into the paper at left is looking down from above at the right. On the right-hand side, we illustrate the sutured manifold resulting from decomposing along the horizontal compressing disks labeled 1 through 8 . Since these disks cut $E(\Sigma)$ into a single 3-ball, and the 

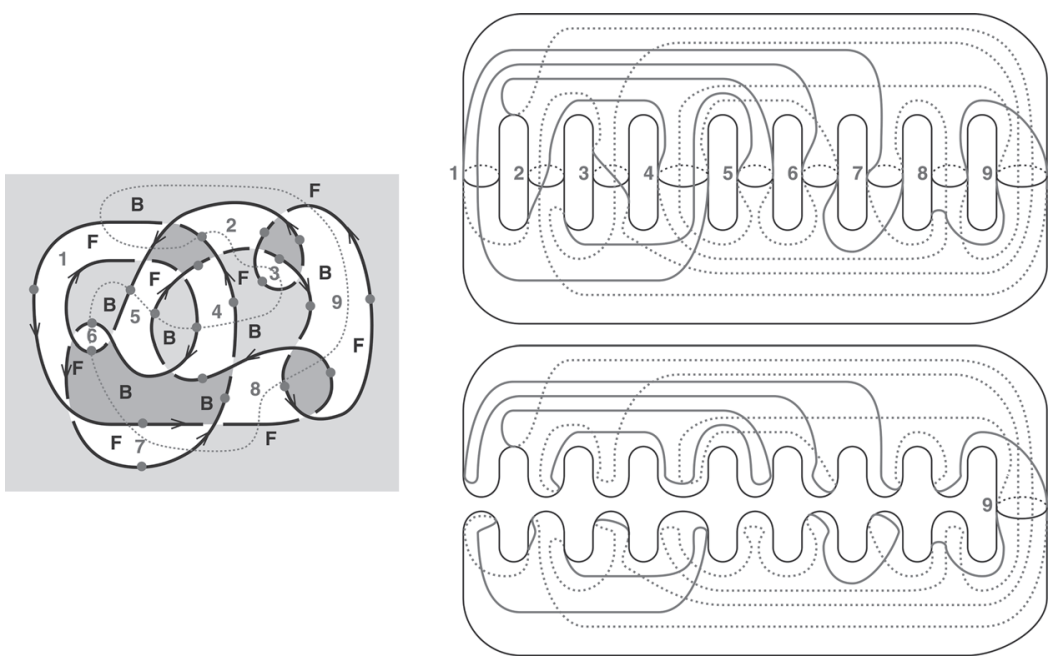

Figure 3: Sutured handlebodies.

suture under decomposition becomes a single curve, the decomposition is taut, so the canonical Seifert surface has minimal genus. A similar sequence of images can be built for the other example given in figure 4; we leave this for the interested reader.

The conditions described by the theorem, and the construction of $K_{n}$ from $K$, are as in figure 5. In the discussion to follow, we assume for ease of exposition that the half-twisted band of the theorem joins a pair of Seifert disks that are not nested, i.e., the disks lie in the same plane and are disjoint. This is not really an issue; every canonical Seifert surface is isotopic to a checkerboard surface [12], and the isotopy process may be assumed to leave fixed all crossings of our original diagram. The idea is that the isotopy to

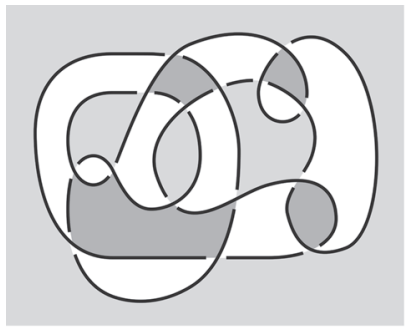

15100154

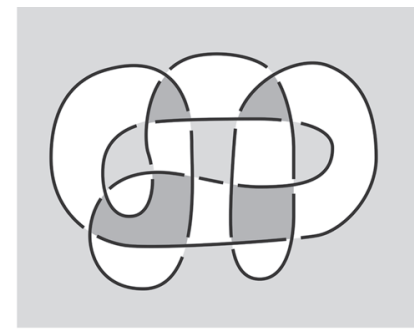

15167945

Figure 4: Stoimenow's examples. 

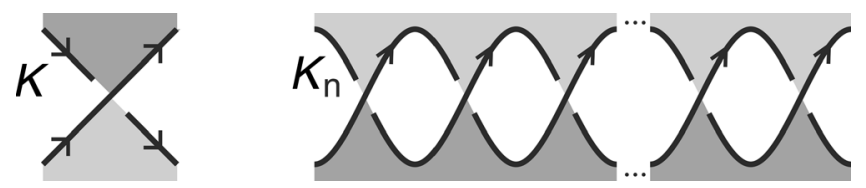

Figure 5: $K$ to $K_{n}$.

a checkerboard is carried out inductively on the outermost disk of a nested collection, as in figure 6; this process adds, but does not delete, crossings to the underlying diagram. The crossing hypothesized by the theorem is therefore still available to us in a checkerboard configuration.

To prove our claim that $g_{c}\left(K_{n}\right)=g_{c}(K)+n$, we show that the canonical Seifert surface $\Sigma_{n}$ for $K_{n}$ built from the diagram $D$ of the theorem admits a taut sutured manifold decomposition. Since each additional pair of crossings introduced does not increase the number of Seifert circles for $\Sigma_{n}$, the Euler characteristic decreases by two, so the genus increases by one, each time.

Since $\Sigma$ is a genus-minimizing Seifert surface for $K,(E(\Sigma), \gamma)$ admits a taut sutured manifold decomposition. To show that $\Sigma_{n}$ is genus-minimizing for $K_{n}$, it suffices to show that there is a sequence of decomposing surfaces taking $\left(E\left(\Sigma_{n}\right), \gamma_{n}\right)$ to $(E(\Sigma), \gamma)$; the taut sutured manifold hierarchy for $(E(\Sigma), \gamma)$ can then be appended to this sequence to give a taut hierarchy for $\left(E\left(\Sigma_{n}\right), \gamma_{n}\right)$. And to do this, it suffices to find a sequence taking $\left(E\left(\Sigma_{n}\right), \gamma_{n}\right)$ to $\left(E\left(\Sigma_{n-1}\right), \gamma_{n-1}\right)$. But this in turn is not difficult; the pair of compressing disks for the pair of 1-handles dual to the pair of half-twisted bands added to obtain $E\left(\Sigma_{n}\right)$ from $E\left(\Sigma_{n-1}\right)$ provide the necessary decomposing surface. On the level of sutured manifolds, this is illustrated in figure 7 . The basic idea is that since the disks "look" as if they belong in the exterior of the checkerboard surface of an alternating knot, the arguments of [7] ensure that the
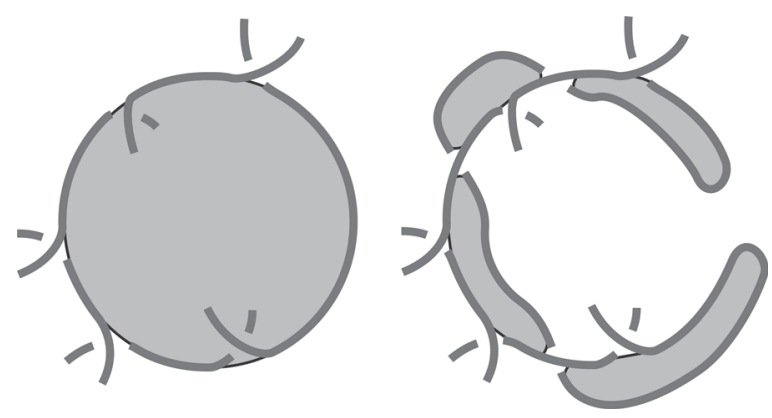

Figure 6: Making canonical surfaces checkerboard. 


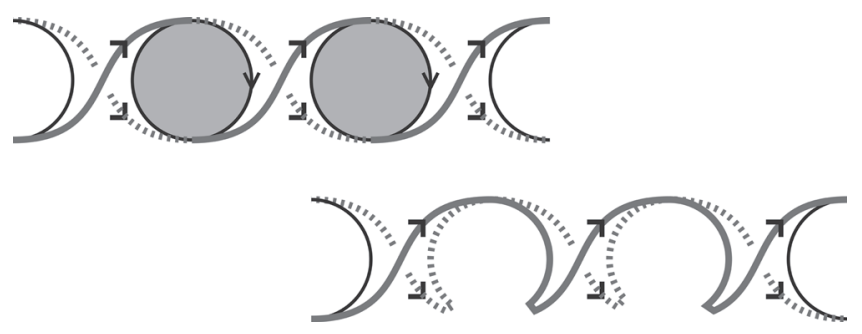

Figure 7: Hierarchies: The inductive step.

sutured manifold obtained by decomposing along them is also the exterior of a Seifert surface; this surface is $\Sigma_{n-1}$.

By induction, therefore, we have $E\left(\Sigma_{n}\right)$ admits a taut sutured manifold hierarchy, and so, by induction, $g\left(K_{n}\right)=g\left(\Sigma_{n}\right)=g(\Sigma)+n$, as desired.

\section{The degree of the HOMFLYPT polynomial is too low}

In this section, we finish the proof of our main theorem by showing that if $K$ is as in the theorem, then $M\left(K_{n}\right)<2 g_{c}(K)+2 n$. We then have $M\left(K_{n}\right)<$ $2 g_{c}(K)+2 n=2 g_{c}\left(K_{n}\right)$, as desired. The needed inequality follows directly from the hypotheses of the theorem and an induction argument using the skein relation satisfied by the HOMFLYPT polynomial.

In what follows, we assume that the crossing given in the hypotheses of the theorem is a positive crossing for $K$ and negative for $K^{\prime}$. A nearly identical argument applies to the reverse situation. To avoid a possible confusion of notation, we will denote by $L_{n}$ the link obtained by replacing the (positive) crossing $c$ with $n$ positive crossings joining the same two Seifert circles as $c$ does, in parallel. (So, in our theorem, $K=L_{1}$ and $K_{n}=L_{2 n+1}$.) It then suffices to show that $M\left(L_{n}\right)<2 g_{c}(K)-1+n$, since then $M\left(K_{n}\right)=$ $M\left(L_{2 n+1}\right)<2 g_{c}(K)-1+(2 n+1)=2 g_{c}(K)+2 n$.

Now by hypothesis, $g_{c}\left(K^{\prime}\right)<g_{c}(K)$. But by Morton's inequality, $M\left(K^{\prime}\right) \leq 2 g_{c}\left(K^{\prime}\right)$, so $M\left(K^{\prime}\right)<2 g_{c}(K)$. From the skein equation for the HOMFLYPT polynomial, $v^{-1} P_{K_{+}}-v P_{K_{-}}=z P_{K_{0}}$, we can immediately conclude that, since the degree of the sum of two polynomials is no more than the maximum of their degrees,

$M\left(K_{0}\right) \leq \max \left\{M\left(K_{+}\right), M\left(K_{-}\right)\right\}-1, \quad M\left(K_{+}\right) \leq \max \left\{M\left(K_{-}\right), M\left(K_{0}\right)+1\right\}$

and

$$
M\left(K_{-}\right) \leq \max \left\{M\left(K_{+}\right), M\left(K_{0}\right)+1\right\}
$$


So using $K_{+}=K=L_{1}, K_{-}=K^{\prime}$ and $K_{0}=L_{0}$, we have

$$
M\left(L_{0}\right) \leq \max \left\{M(K), M\left(K^{\prime}\right)\right\}-1<2 g_{c}(K)-1
$$

This together with $M\left(L_{1}\right)=M(K)<2 g_{c}(K)=2 g_{c}(K)-1+1$ gives us the beginnings of our induction.

We proceed by complete induction. Assume that $n \geq 2$ and $M\left(L_{j}\right)<$ $2 g_{c}(K)-1+j$ for all $j<n$. Then looking at any one of the $n$ parallel crossings $c$ of our diagram for $K_{n}$, we have $K_{+}=L_{n}, K_{-}=L_{n-2}$ and $K_{0}=$ $L_{n-1}$. Then

$$
\begin{aligned}
M\left(K_{+}\right)= & M\left(L_{n}\right) \leq \max \left\{M\left(K_{-}\right), M\left(K_{0}\right)+1\right\} \\
= & \max \left\{M\left(L_{n-2}\right), M\left(L_{n-1}\right)+1\right\}<\max \left\{2 g_{c}(K)\right. \\
& \left.-1+n-2,\left(2 g_{c}(K)-1+(n-1)\right)+1\right\}=2 g_{c}(K)-1+n,
\end{aligned}
$$

as desired. Since the inequality is true for $n=0,1$, by complete induction, $M\left(L_{n}\right)<g_{c}(K)-1+n$ for all $n \geq 0$. This finishes the proof of Theorem 1 .

We now prove Corollary 1.2 by demonstrating that Stoimenow's examples satisfy the hypotheses of the theorem. The sutured manifold calculations of Section 2 verify that $g(K)=g_{c}(K)=4$ for both knots. A routine calculation shows that the HOMFLYPT polynomial of $K=15_{100154}$ is $\left(v^{2}+6 v^{-2}\right) z^{6}+\left(-v^{4}+4 v^{2}+6-5 v^{-2}+v^{-4}\right) z^{4}+\left(-3 v^{4}+4 v^{2}+10-9 v^{-2}\right.$ $\left.+2 v^{-4}\right) z^{2}+\left(-2 v^{4}+v^{2}+6-5 v^{-2}+v^{-4}\right)$ and for $K=15_{167945}$ is

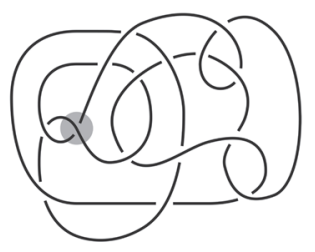

$15_{100154}$

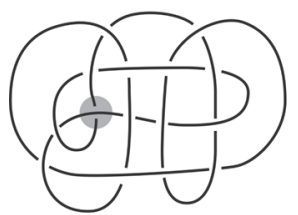

15167945
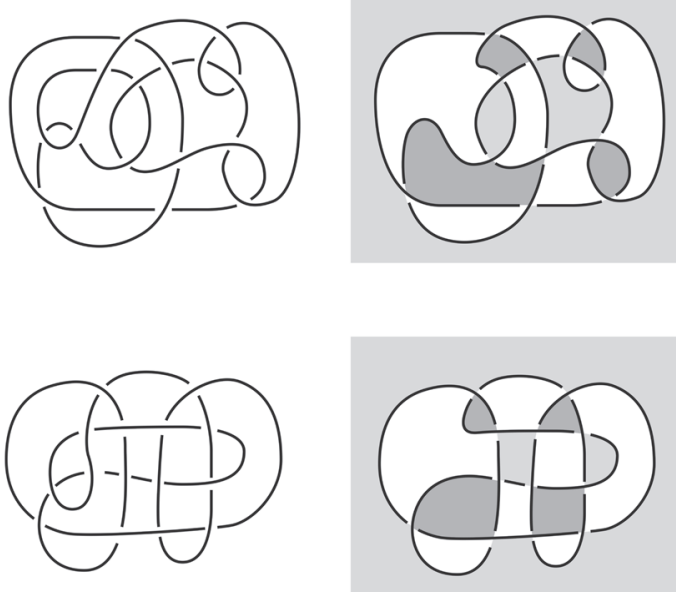

Figure 8: Explicit families of examples. 
$\left(v^{2}+v^{-2}\right) z^{6}+\left(-v^{4}+2 v^{2}+9-4 v^{-2}-2 v^{-4}\right) z^{4}+\left(-2 v^{4}+12-6 v^{-2}-v^{-4}\right.$

$\left.+v^{-6}\right) z^{2}+\left(-v^{4}-v^{2}+6-3 v^{-2}\right)$. In addition, each of the $g_{c}$-minimizing knot diagrams in figure 4 has at least one crossing change which lowers the canonical genus; see figure 8 for canonical surfaces of genus 3 . The relevant crossings join coplanar disks; both Seifert surfaces are checkerboards. Therefore all of the hypotheses of Theorem 1.1 are satisfied, and so each knot gives rise to a family of knots $K_{n}$, for $n \geq 1$, with $g\left(K_{n}\right)=g_{c}\left(K_{n}\right)=n+3$ and $M\left(K_{n}\right)<2 g_{c}\left(K_{n}\right)$. This proves the corollary.

\section{Further considerations}

The main result of this paper was, in some sense, a by-product of our investigations [1] into the canonical genus of Whitehead doubles of knots, motivated by the work of Tripp [24] and Nakamura [15]. (In what follows, we use the term "the Whitehead double" loosely here; in these investigations the resulting calculations are the same no matter how many twists the double has, unless $K$ is the unknot.) If $W(K)$ is the Whitehead double of the knot $K$, and $c(K)$ is the minimal crossing number for $K$, then a direct construction of a canonical Seifert surface for $W(K)(K \neq$ unknot $)$ yields the inequality $g_{c}(W(K)) \leq c(K)$. Together with Morton's inequality we then have

$$
M(W(K)) \leq 2 g_{c}(W(K)) \leq 2 c(K) .
$$

In the cases covered by the papers $[1,15,24]$, an induction argument establishes that $M(W(K))=2 c(K)$, proving that $g_{c}(W(K))=c(K)$ and (as a by-product) $M(W(K))=2 g_{c}(W(K))$.

A posteriori, this method of proof requires that Morton's inequality be an equality. Since we were aware of this prior to beginning our investigations in [1], we were led first to investigate the possible failure of Morton's "equality", which led us to the examples of Stoimenow and so to our own. It is not too difficult to generate examples of knots $K$ for which $M(W(K))<2 c(K)$; the knots $K=8_{19}, 8_{20}$ were the first that the authors found, with the aid of Mathematica and the software package KnotTheory [13]. (In fact, this inequality is strict for every non-alternating pretzel knot $K$ [1].) It follows that for these examples either $M(W(K))<2 g_{c}(W(K))$ or $g_{c}(W(K))<$ $c(K)$. (Exactly one of these is true, for each of the above two knots.) The question is: which of the two inequalities is strict? From the authors' point of view, strictness of the second one would probably be the more interesting.

We are led to believe that Morton's inequality is the one which we would expect to be an equality. This is supported by the known classes of knots for 
which it is true, mentioned in the introduction. It is further supported by a result of Stoimenow [23, Theorem 11.1]; he shows that, asymptotically, as the genus is held fixed but the number of crossings goes to infinity, almost all canonical Seifert surfaces for a link diagram achieve the underlying link's genus, which in turn is equal to the degree of the Alexander polynomial. Since, in terms of the HOMFLYPT polynomial, the Alexander polynomial $\Delta_{K}(t)=P_{K}\left(1, t^{1 / 2}-t^{-1 / 2}\right)$, the $z$-degree of the HOMFLYPT polynomial is at least $2 \cdot \operatorname{deg} \Delta_{K}(t)=2 g_{c}(K)$, in this case. Morton's inequality is therefore an equality.

It seems remarkable that the HOMFLYPT polynomial, which can be computed from any projection of a knot $K$, can be so good at predicting the canonical genus of $K$, and therefore, in some sense, be so good at predicting what the "simplest" projection of $K$ looks like (from the point of view of Seifert's algorithm). A better understanding of when the HOMFLYPT polynomial fails to do so, that is, when Morton's inequality is strict, could help us to better understand why it is usually so good at it. For example, when the inequality is strict, can one always find a skein tree diagram in which every branch of the tree exhibits an unexpected drop in degree as we progress to a collection of unlinks? Or do some knots, in the course of the calculation of $P_{K}(v, z)$ from a diagram, always have an unexpected collision of high $z$-degree terms which fortuitously cancel one another out?

We should mention that there is the potential for a completely different path to families of knots for which Morton's inequality is strict, using connected sums. It is a straighforward calculation that the $z$-degree of the HOMFLYPT polynomial is additive under connected sum, and one might logically expect that the canonical genus is also additive under connected sum, as other knot genera such as the genus [19] and free genus [17] are. But this is not yet known to be true. Were it true, then the connected sum of any knot with a knot for which Morton's inequality is strict would also yield a knot with strict inequality.

Finally, we should point out that we can, in principle, determine if Morton's inequality is an equality for any given knot $K$. This is because we can recursively construct all knots having a canonical Seifert surface up to a given genus $g$; all such surfaces are isotopic to checkerboard surfaces, and fall into finitely-many twist-equivalent classes [21]. HOMFLYPT polynomials within each class are related to one another via the skein relation, and so we can quickly narrow our search down to finitely-many knots having at most a given canonical genus and with the same HOMFLYPT polynomial as $K$. We can then use the solution to the homeomorphism problem for knot complements $[11,25]$ to check each candidate against $K$. This is, of 
course, an extremely laborious process. But this might be worthwhile to carry out for a single knot like $W\left(8_{19}\right)$ or $W\left(8_{20}\right)$, where we know that the canonical genus is at most 8 (by construction) and at least 7 (by Morton's inequality). Perhaps the best that we could hope for is that no knot with canonical genus 7 has the same HOMFLYPT polynomial as $W\left(8_{19}\right)$ and/or $W\left(8_{20}\right)$. Then we would not need to test for equivalence of knots; Morton's inequality would necessarily be the inequality which is strict.

\section{Acknowledgments}

The first author wishes to thank the Department of Mathematics of the City College of New York for their hospitality while a part of this work was carried out. The second author wishes to thank the Department of Mathematics of the University of Nebraska, Lincoln and the Nebraska IMMERSE program for their hospitality and support. The research by M.B. was supported in part by NSF grant \# DMS-0306506 and the research by J.J. was supported in part by NSF grant \# DMS-0354281.

\section{References}

[1] M. Brittenham and J. Jensen, Canonical genus and the Whitehead doubles of arborescent knots, Preprint, 2008.

[2] M. Brittenham, Free Seifert surfaces and disk decompositions, Math. Z. 240 (2002), 197-210.

[3] P. Cromwell, Homogeneous links, J. London Math. Soc. 39 (1989), $535-552$.

[4] R. Crowell, Genus of alternating link types, Ann. of Math. 69 (1959), $258-275$.

[5] P. Freyd, J. Hoste, W. Lickorish, K. Millett, A. Ocneanu and D. Yetter, A new polynomial invariant of knots and links, J. Bull. Amer. Math. Soc. 12 (1985), 239-246.

[6] D. Gabai, Foliations and the topology of 3-manifolds, J. Differential Geom. 18 (1983), 445-503.

[7] D. Gabai, Genera of the alternating links, Duke Math. J. 53 (1986), 677-681.

[8] D. Gabai, Foliations and genera of links, Topology 23 (1984), 381-394. 
[9] H. Goda, A construction of taut sutured handlebodies which are not disk decomposable, Kobe J. Math. 11 (1994), 107-116.

[10] H. Gruber, On knot polynomials of annular surfaces and their boundary links, Preprint (2008).

[11] G. Hemion, On the classification of homeomorphisms of 2-manifolds and the classification of 3-manifolds, Acta Math. 142 (1979), $123-155$.

[12] M. Hirasawa, The flat genus of links, Kobe J. Math. 12 (1995), 155-159.

[13] KnotTheory Mathematica package, available for download from http://katlas.math.toronto.edu/wiki/The_Mathematica_Package_Knot Theory.

[14] K. Murasugi, On the genus of the alternating knot, I,II, J. Math. Soc. Japan 10 (1958), 94-105, 235-248.

[15] T. Nakamura, On the crossing number of 2-bridge knot and the canonical genus of its Whitehead double, Osaka J. Math. 43 (2006), 609-623.

[16] H. Morton, Seifert circles and knot polynomials, Math. Proc. Cambridge Philos. Soc. 99 (1986), 107-109.

[17] M. Ozawa, Additivity of free genus of knots, Topology 40 (2001), 659-665.

[18] J. Przytycki and P. Traczyk, Invariants of links of Conway type, Kobe J. Math. 4 (1988), 115-139.

[19] D. Rolfsen, Knots and links, Publish or Perish Press, Wilmington, DE, 1976.

[20] H. Seifert, Über das Geschlecht von Knoten, Math. Annalen 110 (1934), 571-592.

[21] A. Stoimenow, Knots of genus one or on the number of alternating knots of given genus, Proc. Amer. Math. Soc. 129 (2001), 2141-2156.

[22] A. Stoimenow, On the crossing number of positive knots and braids and braid index criteria of Jones and Morton-Williams-Franks, Trans. Amer. Math. Soc. 354 (2002), 3927-3954.

[23] A. Stoimenow, Knots of genus 2, Preprint, 2003. 
[24] J. Tripp, The canonical genus of Whitehead doubles of a family torus knots, J. Knot Theory Ramifications 11 (2002), 1233-1242.

[25] F. Waldhausen, Recent results on sufficiently large 3-manifolds, Algebraic and geometric topology (Proc. Sympos. Pure Math., XXXII), American Mathematical Society, Providence, RI, 1978, pp. 21-38.

Department of Mathematics

UNIVERSITY OF NEBRASKA

203 Avery HaLL

LINCOLN, NE 68588-0130

USA

E-mail address: mbrittenham2@math.unl.edu

Department of Mathematics and Statistics

Sam Houston State University

Huntsville, TX 77341

USA

E-mail address: jensen@shsu.edu

Received October 11, 2006 